\title{
Nephrotic Syndrome in Children - Studies from South Africa
}

\author{
Gertruida van Biljon \\ University of Pretoria, Department of Paediatrics \\ South Africa
}

\section{Introduction}

Worldwide research has shown that racial differences occur with regard to the histological subtypes, response to treatment and outcome of idiopathic nephrotic syndrome (INS) in children (Bhimma et al. 2006:1847; Bhimma, R. 2009:15; Ingulli \&Tejani 2001:393). Several reasons have been suggested to explain these differences such as a higher prevalence of infections, lower socio-economic status and inequalities in access to health care resources, genetics, and environmental factors but none of these have been substantiated by data. Results from the International Study of Kidney Disease in Children (International Study of Kidney Disease in Children ISKDC 1978:159) showed that the majority of children with INS have minimal change nephrotic syndrome (MCNS) which responds to corticosteroid treatment and that a kidney biopsy is not indicated. Based on these findings empiric corticosteroid treatment was recommended, without performing a kidney biopsy. The study population consisted of predominantly white children from North America, Europe and Asia. These recommendations have been implemented worldwide as standard of care for the past 40 years despite the lack of prospective renal biopsy studies to substantiate this recommendation and which may not be applicable to other settings with a predominance of black patients. An increasing incidence of focal segmental glomerulosclerosis (FSGS) in children and adults with INS has been reported recently (Borges et al. 2007:1309; Filler et al. 2003 :1107; Srivastava et al. 1991:13). Studies reporting the outcome of INS associated with FSGS are variable, which is not surprising, as differences in the population mix, aetiology, pathogenesis and duration of disease are often not taken into consideration. This begs the question whether the recommendation of the International Study of Kidney Disease in Children for the management of INS should still be adhered to. Or should it be revised taking into consideration different racial groups? On the other hand, in the light of the rising incidence of FSGS, it may be prudent to withdraw the recommendation.

Paediatric nephrologists from developing countries, and specifically Africa, need to formulate guidelines specific to their patients with INS. To this end, relevant clinical characteristics such as the antenatal and family history, birth weight, feeding and nutrition, growth and onset of disease should be documented and analysed in their reports. Low birth weight (LBW) which has been shown to be associated with decreased glomerular endowment (Manalich et al. 2007:770, Vehaskari, VM. 2007:490) and subsequent increased risk for the development of chronic kidney disease (CKD), is a case in point, since it is more common in impoverished population groups living in Africa. However, most publications describing the influence of LBW on CKD come from developed countries. (Teeninga et al. 2008:1615) 
Limited resources are often the stumbling block for clinicians in developing countries. For the standard care of a child with INS the minimum investigations necessary in the work-up include urine biochemistry, urine microscopy and investigations to exclude infectious and immune disorders. A kidney biopsy is recommended for all children and should include light microscopy, immuno-histochemistry or immunofluorescent studies and electron microscopy. These investigations are costly, but essential to make a definitive diagnosis. Without a specific diagnosis of the underlying pathology and the associated complications it will not be possible to make reliable recommendations for targeted treatment in a child with INS in a developing country.

\section{Aim}

The aim of the study is to describe the clinical characteristics, histological subtypes, response to treatment and outcome of children with INS treated at the paediatric renal unit of the Steve Biko Academic Hospital (SBAH). This is a level 3 South African hospital, affiliated to the University of Pretoria and the referral centre for the surrounding multiracial population of roughly 5 million children under the age of 14 years.

\section{Methods}

A retrospective audit was performed of consecutive children admitted with a clinical diagnosis of INS. The latter was defined as $\geq 2+$ proteinuria on a urine dipstick test, hypoalbuminaemia of $<25 \mathrm{~g} / \mathrm{L}$ and oedema. Children who had macroscopic haematuria (red or brownish discolouration of urine) substantiated by $>2+$ blood on a urine dipstick test and hypertension in addition to the criteria for INS, were also included and were categorised as nephriticnephrotic. Hypertension was diagnosed according to the $4^{\text {th }}$ Report of the National High Blood Pressure Education Program Working Group on High Blood Pressure in Children and Adolescents. (The Fourth Report of the National High Blood Pressure Education Program Working Group on High Blood Pressure in Children and Adolescents. 2004:555).

The racial groups were documented as black, white, Indian, and mixed. The Indian and white racial groups were pooled and categorised as white. The black and mixed racial groups were similarly pooled and categorised as black. This grouping was done because of known similarities in clinical presentation, response to treatment and outcome in the respective groups. (Bhimma et al.1997:429).

The clinical characteristics analysed were age at presentation, gender, anthropometry (height/length for age and weight for age), and blood pressure.

The following investigations were analysed: urine dipstick tests, urine protein:creatinine ratio $(\mathrm{mg} / \mathrm{mg})$, s-albumin and s-cholesterol, s-creatinine and estimated glomerular filtration rate (eGFR) in $\mathrm{ml} / \mathrm{min} / 1.73 \mathrm{~m} 2$. eGFR was calculated using a modified Schwartz formula (Schwartz. et al. 1987:571), i.e.: [40 x height $(\mathrm{cm}) / \mathrm{s}$-creatinine $(\mu \mathrm{mol} / \mathrm{L})]$. Kidney function at presentation was categorised according to the National Kidney Foundation Kidney Disease Outcomes (K/DOQI clinical practice guidelines 2002:S1). Investigations to rule out secondary nephrotic syndrome include: the third and fourth components of complement (C3 and C4), immunologic tests for systemic lupus erythematosus (antinuclear antibodies and anti-double-stranded DNA antibodies), antistreptolysin $\mathrm{O}$ and anti DNase B titers, hepatitis B and C serology, cytomegalovirus (CMV) antibodies and HIV enzyme-linked immunosorbent assay (ELISA). 
The indications for a kidney biopsy were: a family history of kidney disease, congenital and infantile nephrotic syndrome, children with clinical features suggestive of nephrotic syndrome (NS) other than MCNS, children who failed to respond to an 8-week course of corticosteroids, prior to the administration of cyclophosphamide, children with persisting elevated s-creatinine levels and all black children.

\section{Treatment}

The mainstay of treatment was corticosteroids with the aim to achieve and maintain remission rather than adhering to a standardised protocol. For this reason a higher dose and a longer course were used (daily dose versus alternate day treatment) compared to the ISKDC guidelines (International Study of Kidney Disease in Children 1978:159). The primary contraindications for corticosteroid treatment were children with CNS, secondary NS (e.g. hepatitis B associated nephropathy, Henoch-Schőnlein purpura), children with CKD Stage 3 or more associated with stunting and wasting, cardiomegaly, anaemia and bone mineral disease and presumably immune-compromised children.

Second line immunosuppressive therapy was initiated in a selective group of children, influenced by compliance and socio-economic factors, and only after parental consent had been obtained. Indications for second line immunosuppressive therapy included children with steroid dependent or frequently relapsing NS, those who developed secondary steroid resistance and a selective group of children with partial response. Exclusion criteria for cyclophosphamide treatment included children with underlying chronic infections, e.g. untreated Mycobacterium tuberculosis or HIV infection and those at increased risk of developing acute or chronic infections due to poor nutritional status.

\subsubsection{Corticosteroid treatment}

Prednisone was administered as a single daily dose at $2 \mathrm{mg} / \mathrm{kg} /$ day and tapered to 1 $\mathrm{mg} / \mathrm{kg} /$ day after 4 weeks in children who responded and achieved remission. Further tapering was only initiated after another 4 weeks and treatment stopped after a total of 20 weeks. For those who had not responded to corticosteroid treatment at a dose of 2 $\mathrm{mg} / \mathrm{kg} /$ day by the 4 th week - which occurred commonly in the event of black children this high dose was continued for a maximum of 8 weeks when the dose was tapered according to the response of the child.

Response to treatment was classified as: remission, partial response and steroid resistant.

Remission was defined as no or trace proteinuria on dipstick test for three consecutive days or urine protein:creatinine ratio of $<0.2 \mathrm{mg} / \mathrm{mg}$. Relapse was defined as proteinuria of $\geq 2+$ on dipstick test for three consecutive days or urine protein:creatinine ratio of $\geq 2.0 \mathrm{mg} / \mathrm{mg}$. Partial response was defined as $\leq 2+$ proteinuria on urine dipstick or urine protein:creatinine ratio $<2.0 \mathrm{mg} / \mathrm{mg}$ after a maximum of 8 weeks of high dose steroid treatment $(2 \mathrm{mg} / \mathrm{kg} /$ day). Steroid resistance was defined as persistent proteinuria $\geq 2+$ and urine protein:creatinine ratio $\geq 2.0 \mathrm{mg} / \mathrm{mg}$ after a maximum of 8 weeks of high dose steroid treatment $(2 \mathrm{mg} / \mathrm{kg} /$ day $)$. Steroid dependence was defined as relapse when the dose of corticosteroid treatment was decreased or within two weeks after stopping it. Frequent relapse was defined as $\geq 2$ relapses per 12-month period.

\subsubsection{Cyclophosphamide treatment}

The total course of oral cyclophosphamide for an individual child was calculated as $168 \mathrm{mg} / \mathrm{kg}$ over 8 or 12 weeks administered as $3 \mathrm{mg} / \mathrm{kg} /$ day for 8 weeks or $2 \mathrm{mg} / \mathrm{kg} /$ day 
for 12 weeks (=168 mg/ kg). Cyclophosphamide tablets are sugar coated and contain $50 \mathrm{mg}$ cyclophosphamide/tablet. It cannot be crushed or divided. The dose to be taken per 7-day week was therefore calculated for each patient and was limited to a maximum dose of $3 \mathrm{mg} / \mathrm{kg} /$ day. Tablets were administered for fewer than seven days per week for smaller patients weighing less than $25 \mathrm{~kg}$ who required $<50 \mathrm{mg} /$ day (i.e. no treatment on weekend days).

A kidney biopsy was performed in all children before cyclophosphamide treatment was initiated. No child received a second course of cyclophosphamide.

\subsubsection{Intravenous methylprednisolone pulse treatment}

A course of intravenous methylprednisolone was administered in three scenarios, i.e. children with anasarca and who were resistant to their first course of oral corticosteroid treatment, children who became steroid dependent or developed frequent relapses after having had a course of cyclophosphamide treatment and children with partial response to oral corticosteroid and cyclophosphamide treatment. Selection of patients for this form of treatment was further influenced by their ability or willingness to frequently return to the hospital and by the absence of recurrent infections.

Intravenous corticosteroid treatment consisted of methylprednisolone $30 \mathrm{mg} / \mathrm{kg} /$ dose (maximum dose of $1000 \mathrm{mg}$ ) administered according to established guidelines (Mendoza et al 1990:303), but excluding cyclophosphamide treatment (Table 1).

It was discontinued when a patient experienced unacceptable adverse effects, e.g. progressive fluid retention or volume overload, with worsening hypertension or when proteinuria remained unchanged after 6 to 8 weeks.

\begin{tabular}{llll}
\hline Week & M-Pa & N & Prednisone \\
\hline $1-2$ & $30 \mathrm{mg} / \mathrm{kg}$ thrice weekly & 6 & None \\
$3-10$ & $30 \mathrm{mg} / \mathrm{kg}$ per week & 8 & $2 \mathrm{mg} / \mathrm{kg} /$ day b \\
$11-18$ & $30 \mathrm{mg} / \mathrm{kg}$ per 2 weeks & 4 & $1.5 \mathrm{mg} / \mathrm{kg} /$ day tapered slowly \\
$19-52$ & $30 \mathrm{mg} / \mathrm{kg}$ per 4 weeks & 8 & $1 \mathrm{mg} / \mathrm{kg} /$ day tapered slowly \\
$53-78$ & $30 \mathrm{mg} / \mathrm{kg}$ per 8 weeks & 4 & $0.5 \mathrm{mg} / \mathrm{kg} /$ day tapered slowly \\
\end{tabular}

$\mathrm{a}=$ Maximum dose $1000 \mathrm{mg}$

$\mathrm{b}=$ Maximum dose $60 \mathrm{mg}$

Table 1. Intravenous methylprednisolone (M-P) pulse regimen

\subsubsection{Adjunctive treatment}

Adjunctive treatment included diuretics for symptomatic management of oedema, antihypertensive drugs, multivitamin and folic acid supplementation, alpha-calcidol for children on high-dose corticosteroid treatment and with persisting nephrotic-range proteinuria. Treatment with an angiotensin converting enzyme inhibitor (ACE-inhibitor) was given for its antiproteinuric effect to alleviate persistent proteinuria and as an antihypertensive drug if needed. Other antihypertensive drugs used included a $\beta$-blocker (atenolol), calcium antagonist (amlodipine), a-blocker (prazosin) and a vasodilator (hydralazine). Angiotensin receptor blockers were not prescribed because of unavailability. 
Diuretics of all classes, namely furosemide, hydrochlorothiazide and spironolactone were used in combinations if needed for children with diuretic-resistant NS. Hydroxymethylglutharyl coenzyme A reductase inhibitors (statins) were selectively prescribed to children older than 5 years with persistent NS associated with an elevated total cholesterol level of $>10 \mathrm{mmol} / \mathrm{L}$. All children received a diet containing reduced salt and saturated fat. Protein intake was not restricted.

\section{Ethical approval}

The study was approved by the University of Pretoria Research Ethics Committee and permission was obtained from the chief executive officer of the hospital to access hospital files.

\section{Statistical analysis}

Categorical data was reported as proportions and quantitative data as means (standard deviations) and medians (range).

Frequencies of variables within groups were compared using the two-sided Fisher exact test and a p-value $<0.05$ was regarded as significant. Renal and patient survival in the two population groups were assessed using Kaplan Meier non parametric life table survival analysis.

\section{Results}

Over a period of 23 years (1986 - 2009) 358 children with a clinical diagnosis of NS were admitted and comprised the study group. Of these $278 / 358$ (77.7\%) were black and 80/358 $(22.3 \%)$ white. The median age was 58 months (range $0.5-144$ months) and the male: female ratio 1.3:1.

The age at presentation was categorised in 4 groups as depicted in table 2 . Twenty seven children were $\leq 12$ months of age at the time of presentation, of whom 15/27 (4\%) presented within the first 3 months of life and were diagnosed with CNS. An additional ten children were also diagnosed with CNS but were referred later, when they were older than 3 months. Fifty percent $(179 / 358)$ of the children were in the age group $>12-72$ months.

\begin{tabular}{lcc}
\hline \multicolumn{1}{c}{ Age category (months) } & Number & $\%$ \\
\hline $0-3$ & 15 & \\
$>3-12$ & 12 & 4.2 \\
$>12-72$ & 179 & 3.3 \\
$>72$ & 152 & 50.0 \\
\hline Total & 358 & 42.5 \\
\hline
\end{tabular}

Table 2. Age Categories of Children at Presentation

Stunting (height/length for age z-score > -2SD) was present in 88/358 (24.6\%) children. Microscopic haematuria was present in 52/109 (47.7\%) and macroscopic haematuria in 34/109(9.5\%) children with MCNS.

A constellation of clinical and laboratory features including, age $>12$ months to 72 months, normal blood pressure, absence of haematuria, normal renal function and normal levels of 
serum $\mathrm{C}_{3}$ have been quoted as suggestive of MCNS with an expected good prognosis (International Study of Kidney Disease in Children 1978:159). In this study 67\% of the children with MCNS fulfilled the criteria for the diagnosis, apart from not having a normal blood pressure. This finding has been described by others (Habib et al 1971) which questions the validity of the criteria for diagnosing MCNS.

Hypertension was present in $227 / 358(63.4 \%)$ children at presentation of whom $171 / 227$ $(47.8 \%)$ required treatment with antihypertensive drugs for at least 6 weeks. Diuretics were used for symptomatic management of oedema and were often used as a first line antihypertensive drug in children considered to have volume overload. Of those with persistent hypertension 146/171 (85.4\%) received an ACE-inhibitor as the preferred antihypertensive drug. Overall 213/358 (59.5\%) received an ACE-inhibitor for its antiproteinuric effect. Renal function and s-potassium were monitored in all children who were treated with an ACE-inhibitor. No child experienced an allergic response or developed significant coughing with this treatment. An acute increase in s-creatinine levels occurred in some patients, usually in association with volume contraction, which was reversible in all cases with fluid resuscitation.

The mean s-albumin level at the time of presentation was $13.2 \pm 5.2 \mathrm{~g} / \mathrm{L}$. Some patients had below detectable s-albumin levels and for these the lowest value documented was an arbitrary level of $10 \mathrm{~g} / \mathrm{L}$ for the purpose of statistical analysis. This means that the true mean level was in fact lower. The mean s-cholesterol at the time of presentation was $11.4 \pm 7.3$ $\mathrm{mmol} / \mathrm{L}$. As already stated above, statins were only prescribed to a limited number of older children with persistent hypercholesterolaemia. The main reason why younger children with similar high cholesterol levels were not treated with statins is the lack of long-term safety information on the effects of these drugs on the developing brain, immune functions, hormones and energy metabolism.

Kidney function was monitored using change in eGFR over time. Despite its limited accuracy, especially in children with poor muscle bulk, it was the only feasible test which could be done at regular intervals at follow-up visits. The results of eGFR at the time of presentation and at last follow up for the two race groups are depicted in table 3.

\begin{tabular}{llllll}
\hline $\begin{array}{l}\text { CKD eGFR } \\
\text { Stage }\left(\mathbf{m l} / \mathbf{m i n} / \mathbf{1 . 7 3 m ^ { 2 }}\right)\end{array}$ & \multicolumn{2}{c}{$\begin{array}{c}\text { eGFR at presentation } \\
\mathbf{n}=\mathbf{3 5 8}\end{array}$} & \multicolumn{2}{c}{$\begin{array}{c}\text { eGFR last follow up } \\
\mathbf{n}=\mathbf{3 5 8}\end{array}$} \\
\hline & & $\begin{array}{c}\text { Black children } \\
\mathbf{n = 2 7 8}\end{array}$ & $\begin{array}{c}\text { White children } \\
\mathbf{n = 8 0}\end{array}$ & $\begin{array}{c}\text { Black children } \\
\mathbf{n = 2 7 8}\end{array}$ & $\begin{array}{c}\text { White children } \\
\mathbf{n}=\mathbf{8 0}\end{array}$ \\
& & $189(68.0 \%)$ & $55(68.8 \%)$ & $178(64.0 \%)$ & $66(82.5 \%)$ \\
$\mathbf{1}$ & $\mathbf{2 9 0}$ & $42(15.1 \%)$ & $20(25.0 \%)$ & $29(10.4 \%)$ & $10(12.5)$ \\
$\mathbf{2}$ & $\mathbf{6 0 - 8 9}$ & $27(9.7 \%)$ & $4(5.0 \%)$ & $18(6.5 \%)$ & $1(1.3 \%)$ \\
$\mathbf{3}$ & $\mathbf{3 0 - 5 9}$ & $20(7.1 \%)$ & $1(1.3 \%)$ & $53(19.0 \%)^{*}$ & $3(3.8 \%)$ \\
$\mathbf{4}+\mathbf{5}$ & $<\mathbf{2 9}$ & &
\end{tabular}

eGFR = Estimated glomerular filtration rate

*One black child with CKD stage 5 who had a successful kidney transplant is included in the number of black children with stage 4 and 5 CKD.

Table 3. eGFR at the Time of Presentation and During Follow Up in the Two Race Groups

Significantly more black compared to white children had stage 4 or 5 CKD on presentation, $7.1 \%$ vs. $1.3 \%$ respectively ( $p=0.03$ ), or had developed stage 4 or 5 CKD at the time of last follow up, $19.0 \%$ vs. $3.8 \%$ respectively $(p=0.000)$. 
A secondary cause for NS was identified in 26 (7.2\%) children including HenochSchőnlein purpura, systemic lupus erythematosus, chronic hepatitis B infection, HIV infection and IgA glomerulonephritis. Ten children $(2.8 \%)$ had chronic hepatitis B associated nephropathy. None of them were given any immunosuppressive treatment, interferon or other specific antiviral treatment. In eleven children a genetic cause of NS was suspected.

CNS was diagnosed in 25/358 (7\%) children, none of whom had a syndromic form of CNS. Investigations for mutations of NPHS1, NPHS2 and WT1 were not done in any of the children with CNS or suspected familial NS because of unavailability.

Kidney biopsies were performed in 318/358 (89\%) children. The main histological diagnoses are depicted in table IV. Eighteen children had inconclusive histology which was reported as "FSGS cannot be excluded." In these cases there was a chronic inflammatory cell infiltrate in the interstitium, interstitial fibrosis and tubulo-interstitial atrophy suggestive of FSGS but the biopsy sample did not have glomeruli with focal sclerosis. If this group of children is added to the group with definite FSGS, the frequency of FSGS increases to 98/318 (31\%). Eleven children (10 black, 1 white) were diagnosed with immune complex glomerulonephritis (ICGN), based on the presence of immune deposits in the basement membrane on electron microscopy examination and another 26 ( 25 black, 1 white) had ICGN with secondary glomerular sclerosis.

\begin{tabular}{lllll}
\hline Histological diagnosis & $\begin{array}{l}\text { White } \\
\text { Number (\%) }\end{array}$ & $\begin{array}{l}\text { Black } \\
\text { Number }\end{array}$ & $\begin{array}{l}\text { Total } \\
(\mathbf{\%})\end{array}$ & p-value \\
\hline & & & & \\
MCNS & $42(66)$ & $67(26.4)$ & $109(34.2)<0.001^{*}$ \\
Focal segmental glomerulosclerosis (FSGS) & $9(14)$ & $71(28)$ & $80(25)$ & $0.01^{*}$ \\
MCNS - FSGS ** & $7(11)$ & $11(4.3)$ & $18(5.7)$ & $0.048^{*}$ \\
Immune complex glomerulonephritis (ICGN) & $1(1.6)$ & $10(4)$ & $11(3.4)$ & 0.31 \\
ICGN and secondary FSGS & $1(1.6)$ & $25(9.8)$ & $26(8.2)$ & $0.02^{*}$ \\
Membranous nephropathy (MN) & $0(0)$ & $14(5.5)$ & $14(4.4)$ & $0.04^{*}$ \\
Mesangiocapillary glomerulonephritis & $1(1.6)$ & $12(5)$ & $13(4)$ & 0.22 \\
Congenital nephrotic syndrome (CNS) & $0(0)$ & $25(9.8)$ & $25(8)$ & $0.02^{*}$ \\
Other & $3(4.7)$ & $19(7.5)$ & $22(7)$ & \\
& & & & \\
Total *** & $64(100)$ & $254(100)$ & $318(100)$ & \\
\hline
\end{tabular}

*Statistically significant

**MCNS-FSGS: Histology was reported as "FSGS cannot be excluded" and was therefore inconclusive but very suggestive of FSGS due to the presence of a chronic inflammatory cell infiltrate in the interstitium, interstitial fibrosis and tubular atrophy on the biopsy.

***Includes all children who had kidney biopsies, including the children with congenital nephrotic syndrome.

Table 4. Main Histological Subtypes of Nephrotic Syndrome $(\mathrm{n}=318)$

In table 5. the frequencies of all the histological subtypes excluding CNS are depicted, which is in line with the procedure followed by the ISKDC. CNS is considered a distinct form of NS with a unique etio-pathogenesis and a high frequency of underlying genetic mutations 
and is therefore usually not analysed with other forms of INS. Kidney biopsies were done in 318 children (64 white and 254 black). After exclusion of the 25 children with CNS, all of whom were black, the frequencies of the histopathological subtypes of only the remaining 229 black children changed.

\section{Histological diagnosis}

MCNS

Focal segmental glomerulosclerosis (FSGS)

MCNS - FSGS **

Immune complex glomerulonephritis (ICGN)

ICGN and secondary FSGS

Membranous nephropathy

Mesangiocapillary glomerulonephritis

Other

***Total

$\begin{array}{llll}\text { White } & \text { Black } & \text { Total } & \text { p-value } \\ \text { Number }(\%) & \text { Number }(\%) & (\%) & \end{array}$

$42(65.6)$

$67(29.3)$

$109(37.2)<0.000^{*}$

$9(14.0)$

71(31.0)

$80(27.3) \quad 0.004^{*}$

$7(10.9)$

$11(4.8)$

18 (6.1) $\quad 0.07$

$10(4.4)$

$11(3.7) \quad 0.26$

1 (1.6)

25(11.0)

$26(8.9) \quad 0.01^{*}$

$0(0)$

$14(6.1)$

$14(4.8) \quad 0.03^{*}$

1 (1.6)

$12(5.2)$

$13(4.4) \quad 0.18$

$3(4.7)$

$19(8.3)$

$22(7.5)$

*Statistically significant

**MCNS-FSGS: Histology was reported as "FSGS cannot be excluded" and was therefore inconclusive but very suggestive of FSGS due to the presence of chronic inflammatory cell infiltrate in the interstitium, interstitial fibrosis and tubular atrophy on the biopsy.

***All children who had kidney biopsies, but excluding the children with congenital nephrotic syndrome.

Table 5. Histological Subtypes of Nephrotic Syndrome Excluding Children with Congenital Nephrotic Syndrome $(\mathrm{n}=293)$

The incidences of the four major histological subtypes (MCNS, FSGS, membranous nephropathy, mesangiocapillary glomerulonephritis were significantly higher in the black children (Table 5).

\subsection{Results of treatment}

Remission with oral corticosteroid treatment was achieved in 33/41(81\%) white vs $33 / 59(56 \%)$ black children $(\mathrm{p}=0.02)$ who had MCNS. The response rate of the black children is similar to the $60 \%$ response rate in black children reported previously from Kalafong Hospital (Prinsloo JG. 1986:375) but lower than the 78\% response rate reported a decade later in children from the Chris-Hani Baragwanath Hospital (Johannesburg), both tertiary hospitals in South Africa (Thomson 1997:402). Oral corticosteroid treatment resulted in remission in $3 / 9$ white children and $8 / 40$ black children with FSGS $(\mathrm{p}=0.6)$. In the combined group of children with FSGS and MCNS-FSGS 9/13 (69.2\%) white children vs 4/14 (28.5\%) $(\mathrm{p}<0.05)$ black children went into remission with this treatment. Twenty five children who failed to respond to oral corticosteroid treatment were treated with a course of intravenous methylprednisolone of whom only 4 (one white and 3 black children) went into complete remission. This form of treatment was abandoned because of its poor efficacy, high toxicity and cost and the disruptive effect it had on school attendance. Hundred children were 
treated with cyclophosphamide, 46 were white and 54 black. The response rate to this treatment was statistically significantly different in the white and black children. Sustained remission was achieved in $37 / 46(80 \%)$ white and in $23 / 54(43 \%)$ black children ( $\mathrm{P}=0.002$; $95 \%$ CI 2.2 - 13.7). The ISKDC reported no benefit of orally administered cyclophosphamide and prednisone compared to prednisone alone for the treatment of steroid resistant NS (Tarshish, $\mathrm{P}$ et al. 1996: 590). Their report and the poor response of children with steroid resistant NS in this study prompted discontinuation of cyclophosphamide treatment in children with steroid resistant NS since 2007. No patient experienced side effects of cyclophosphamide treatment, but they were all monitored at least every 10 to 14 days throughout the duration of the treatment. Several children who had been in contact with chicken pox were given human varicella-zoster immune globulin and/or acyclovir prophylactically, but none developed serious chicken pox.

\subsection{Morbidity and mortality}

Acute reversible renal failure occurred in 35/358 (9.8\%) children and thrombotic complications, other than strokes in $9 / 358$ (2.5\%). Six children $(1.7 \%)$ developed strokes, one of whom developed bilateral sequential middle cerebral artery thromboses a few months apart. She was one of a family of 3 children who all had steroid resistant NS. At the time that she developed the first stroke she was not dehydrated, but had a mild lower respiratory tract infection, iron deficiency anaemia and a thrombocytosis, which are known risk factors for thrombo-embolic complications in children with nephrotic syndrome.

Fourty eight percent of all children experienced acute invasive bacterial infections, including pneumonia, peritonitis and septicaemia. In those with steroid sensitive NS acute bacterial infections occurred during relapses. The frequency of infection was inversely related to age and was particularly high in children younger than 3 months. Of these children $87 \%$ developed serious infections compared to $39 \%$ of children older than 6 years. Streptococcus pneumoniae was the predominant causal organism, followed by Escherichia coli and other gram negative organisms. Twenty six children $(7.3 \%)$ developed peritonitis of whom three demised due to pneumococcal septicaemic shock. Pneumococcal infections occurred in $7 / 80$ $(8.7 \%)$ white children vs $19 / 278(6.8 \%)$ black children $(p=0.6)$. Until recently pneumococcal polysaccharide vaccine was given to all children younger than 5 years at the time of their first presentation, despite its limited efficacy in children younger than 2 years. Since 2009 the pneumococcal conjugate vaccine is available in South Africa which is used for revaccination of this group of children. Long term prophylactic penicillin was not used.

Chronic hepatitis B infection (positive HBsAg and/or HBeAg) occurred in 10/358 (2.8\%) children, all of whom were black. Hepatitis B vaccine was included in the routine immunization schedule of children in South Africa since 1991 and since that time no child was diagnosed with hepatitis B related NS. No child had hepatitis C related NS. Investigations to rule out CMV infection were only done in children with CNS and in those with atypical clinical features of NS, including anaemia, hepatosplenomegaly, skin rash or positive central nervous system signs. In most cases only CMV IgM and IgG were done, which were often both positive due to unexplained reasons at the time, because the test for CMV viral load was not available. Should a CMV infection be diagnosed it is not necessarily proof of the causality of the NS.

All children were screened for underlying Mycobacerium tuberculosis infection with a chest X-ray, gastric aspirates or induced sputum cultures and Mantoux test (skin prick test with intradermal injection of purified protein derivative of Mycobacterium tuberculosis). Because 
children uncommonly have sputum positive tuberculosis, several of our patients received empiric anti-tuberculous treatment for 6 months when the diagnosis could not be unequivocally excluded. A high prevalence of tuberculosis associated with a deleterious effect on renal function was reported in black children with FSGS. (Kala et al.1993:392). It has been postulated that immune responses mediated by infections with Mycobacterium tuberculosis and HI-virus may contribute to glomerulosclerosis. Mycobacterium tuberculosis infection was present in too few patients in this study to draw any conclusion. Investigations for HIV infection were only done in children who had clinical features suggestive of the disease and whose parents had given consent to testing. It is therefore not possible to report on the true incidence of NS associated with HIV infection. Patients with HIV infection had a variety of histological lesions, including, immune complex glomerulonephritis, immunotactoid and fibrillary glomerulonephritis and FSGS. No patient had HIV collapsing glomerulopathy which has been reported as one of the commonest histological lesions in black adult patients with HIV infection. Recently HIV- associated kidney disease has been reported to have become the most common form of kidney disease in children seen in the renal unit at one of the academic hospitals in South Africa. (Bhimma R, 2009:15)

Only one infant with CNS had congenital syphilis and treatment with penicillin did not result in cure of the disease. Chronic "quartan malarial nephropathy" or other parasitic related forms of NS did not occur.

\subsection{Outcome}

At presentation $21 / 358$ children had CKD stage 4 or 5 . Of the black children $20 / 278$ (7.2\%) had CKD stage 4 or 5 compared to $1 / 80(1.3 \%)$ white children $(p=0.03)$. Over the period of follow up more black children (53/278) (19\%) developed stage 4 or 5 CKD compared to white children $(3 / 80)(3.8 \%)(\mathrm{p}=0.000)$. Kaplan-Meier estimation of renal survival depicting the difference in renal survival in black and white children is demonstrated in Fig1. One black child was successfully transplanted during the follow up period and had normal renal function at last follow up. Persistent nephrotic range proteinuria is associated with a rapid progression to end-stage kidney disease. Several children in this study had long standing suboptimal management of nephrotic range proteinuria when presenting to the SBAH which contributed to a more rapid progression to end stage kidney disease.

Forty three patients died during the follow up period. Three $(3.7 \%)$ white children died of whom 2 succumbed to complications of renal failure (renal deaths) and one died due to pneumococcal septicaemia (non renal death). Forty black children died during the follow up period, mostly due to end stage renal failure. In the black children infectious related deaths occurred mostly in the children with congenital NS. Black children had a significantly higher mortality compared to white children $(40 / 268$ vs. $3 / 80)(\mathrm{p}<0.001)$. Kaplan-Meier patient survival estimate depicting the difference in patient survival for black and white children is demonstrated in Fig 2.

\section{Discussion}

This study population differs in several aspects from those reported from developed countries. The majority of patients are black with an inherent risk of CKD due to a genetic predisposition aggravated by poor socio-economic circumstances and chronic infections. Poor prognostic indicators namely stunting, profound hypoalbuminaemia, long standing nephrotic-range proteinuria, hypertension and impaired kidney function are common at presentation against a background of tuberculosis and HIV infection. 


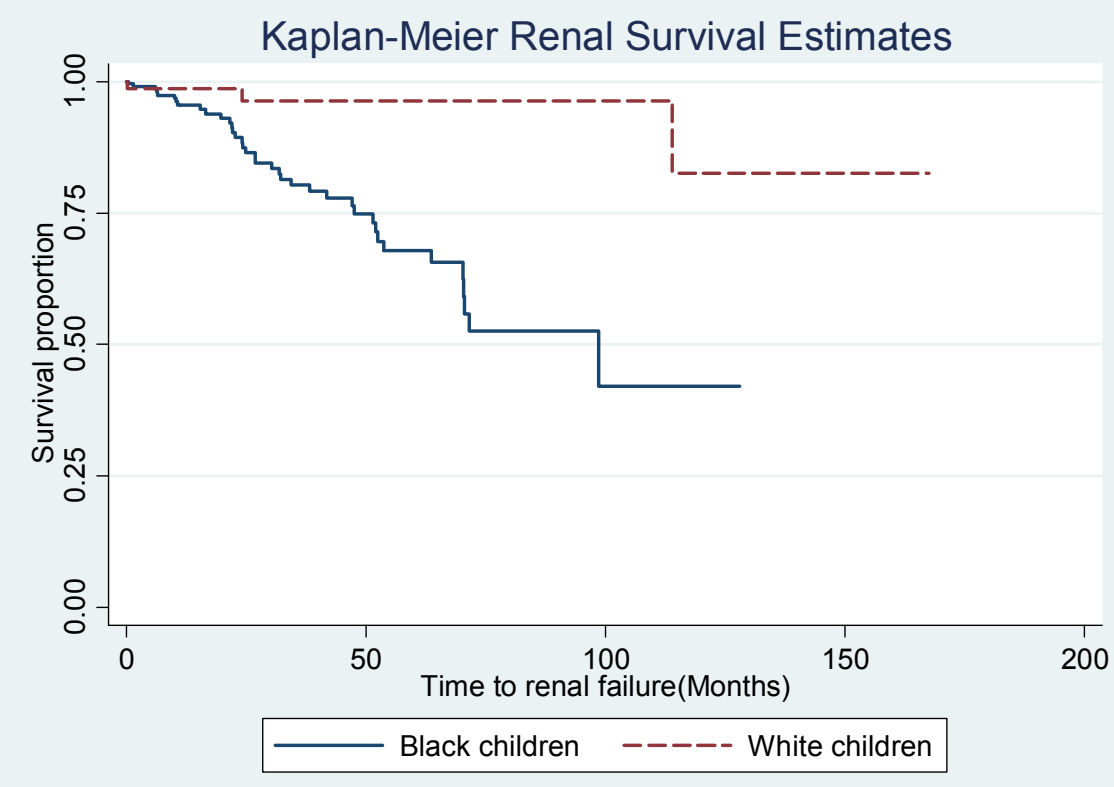

Fig. 1. Kaplan-Meier estimation of renal survival

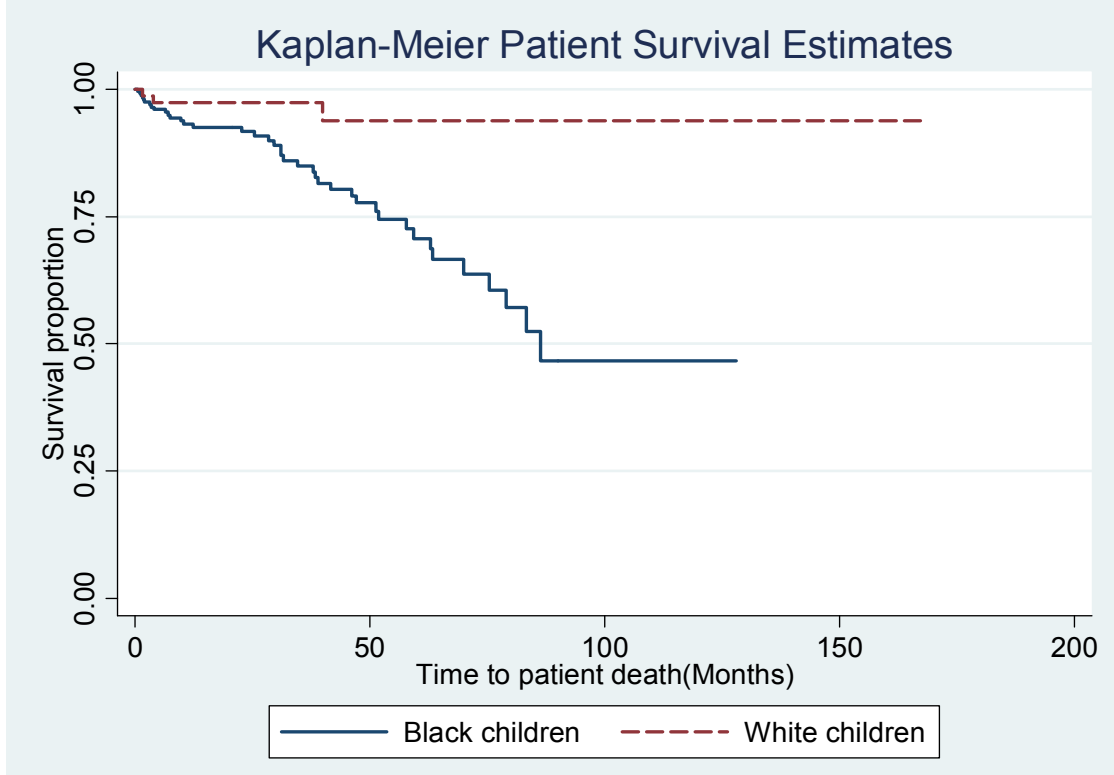

Fig. 2. Kaplan-Meier estimation of patient survival 
The results of this audit confirm that significant racial differences exist in the clinical presentation, histological subtypes, response to treatment and outcome in South African children with NS. Similar findings have been reported by other study groups in South Africa (Bhimma et al. 2006: 1847; Bhimma R, 1997: 429), from other countries in Africa (Yao Doe, J. et al. 2006: 672; Olowu et al. 2010:200) and also from elsewhere in the world (Ingulli, E. \& Tejani, A. 2001:393). In their study on idiopathic FSGS in children Ingulli et al. (Ingulli, E. \& Tejani, A. 2001:393) reported that the rate of FSGS was higher (32.2\% vs. 20\%), and progress to end stage kidney disease was more common (78\% vs. 33\%) in black and Hispanic compared to white children. Olowu et al. (Olowu et al. 2010:200) reported an incidence of $18.5 \%$ MCNS and 25.9\% FSGS in their study of black Nigerian children with INS. Ethnic differences in the incidences of FSGS have now been well established. Over the past 10 years there have been several reports of an increasing incidence of FSGS in children (Borges et al. 2007:1309; Filler et al. 2003: 1107)

In this study $77 \%$ of the children were black and $23 \%$ were white. There was a significant difference in the incidences of the main histological subtypes (MCNS, FSGS, immune complex glomerulonephritis, secondary FSGS and membranous nephropathy) between black and white children. The incidence of MCNS was $65.6 \%$ and $29.3 \%(p<0.000)$ and of FSGS, $14 \%$ and $31 \%(p=0.004)$ in white and black children respectively.

The first course of corticosteroid treatment was often longer or more intense than that recommended by the ISKDC (International Study of Kidney Disease in Children: 1978:13) because of delayed achievement of remission or only partial response after the first four weeks. Corticosteroid treatment was also often given daily rather than on alternate days because parents failed to understand alternate day dosing schemes. White children with MCNS had a better response to oral corticosteroid treatment compared to black children with MCNS ( $80 \%$ vs 56\%; p=0.02). Remission with oral corticosteroid treatment was achieved in more white children with FSGS compared to black children with FSGS, although this difference did not reach statistical significance ( $33 \%$ vs $20 \%$; p $=0.6)$. Response rates in both race groups in this study were much lower compared to the $93.1 \%$ response rate reported by the ISKDC (International Study of Kidney Disease in Children: 1978:13).

After 2000 we stopped using high dose intravenous methylprednisolone for the treatment of steroid resistant NS in our patients for several reasons. It is too costly, it places a heavy burden on the family and child, and only 4 of 25 children (16\%) went into remission with this treatment. This very poor response rate compared to that of Mendoza et al (Mendoza, S.A. et al. 1990:303), who reported complete remission in 52\% of their patients, can possibly be explained by the fact that we omitted an alkylating agent in our treatment regimen. Adhikari et al (Adhikari et al. 1997:423) reported a dismal outcome in 12 South African children with FSGS who were treated with a combination of intravenous methylprednisolone and an alkylating agent. Although they considered this treatment as "promising," their patients developed serious side effects including alopecia, cataracts, leukopaenia, systemic candidiasis, gram negative septicaemia, and one child demised of a serious infection which was undoubtedly caused by the severe immune suppression associated with the treatment.

Infections remain a serious risk to all children during a relapse of NS, which is practically always for those with steroid resistant NS. This risk is intensified in those living in poor socio-economic circumstances. Forty eight percent of our patients experienced acute invasive bacterial infections and three succumbed to documented pneumococcal 
septicaemia. Management of the children with CNS was particularly challenging, due to their serious immune compromised state and high frequency of recurring infections. All children with CNS were black and screening for a possible infectious cause was not very fruitful. Genetic studies were not undertaken in any of our patients as we do not have access to genetic laboratory services. Kidney biopsy revealed idiopathic FSGS or secondary glomerular sclerosis in 15/25 children with CNS. A very dense chronic inflammatory cell infiltrate was present in all cases. Children with CNS were not treated with corticosteroids or other immunosuppressive drugs. Most of them remained in hospital for long periods or required frequent admissions for treatment of bacterial infections or gastroenteritis. An ACE inhibitor was not prescribed to any child less than 3 months old and was usually only given to children older than 12 months.

Because of the lower incidence of MCNS in black children in South Africa first reported in 1979, (Bhimma et al. 1997:429; Lewin, et al. 1979: 88) it has been our practice to biopsy all black children at the time of their first presentation. For the same reason paediatric nephrologists elsewhere in Africa have also advocated pre-treatment renal biopsies in their patients. Olowu et al. (Olowu et al. 2010:200] reported that only 18.5\% of the black children with INS in their study of Nigerian patients had MCNS.

Most centres in developed countries are still following the ISKDC recommendation regarding biopsies despite worldwide reports of an increasing incidence of FSGS in both children and adults. Filler et al (Filler et al. 2003 :1107) reported a declining incidence of MCNS from $81.1 \%$ to $64 \%$ and an increasing incidence of FSGS from 10.8 to $24.7 \%$ of FSGS in their childhood population in Ontario, over two time periods 1985-1993 and 1993-2002. The incidence of MCNS in the first period did not differ significantly from that reported by the ISKDC (International Study of Kidney Disease in Children 1978: 13) and although not specifically reported, the inference is made that the majority of their patients responded to corticosteroid therapy. The incidence of FSGS in their patients has more than doubled over 17 years while the population under study remained stable. Race is not mentioned in their study. Despite an alarming increase in the incidence of FSGS in their patients, they argue that empirical steroid treatment with a cut-off point at 28 days is still justifiable. During the second period of their study the incidence of MCNS is similar to the incidence of MCNS in white children in our study, $64.7 \%$ vs. $65.6 \%$ and the incidence of FSGS slightly less than that of FSGS in black patients in our study $24.7 \%$ vs. $31 \%$.

Already in 1997 Thomson et al. (Thomson, P.D. 1997:508), performing pre-treatment biopsies in all their black patients, reported an incidence of FSGS in 31.3\% which is identical to the $31 \%$ incidence of FSGS in black patients in this study. It appears that there has not been an increase in the incidence of FSGS in black children in Gauteng Province in South Africa over this period.

It can be expected that the HIV epidemic has contributed to the incidence of CKD in South Africa in general, but its possible role in the development of FSGS, or its contribution to an increase in the incidence of FSGS, is uncertain. Local multi-centre prospective research studies in patients with HIV-associated nephropathy will be necessary to explore this question.

Primary FSGS is a spectrum of podocytopathies caused by a variety of contributing etiologies, including genetics, infections, environment, including the intra-uterine environment, drugs and toxins. It is an aggressive disease, more so in black children compared to white children. The rationale of performing a kidney biopsy at presentation is 
that it confirms the histological subtype and may give clues to the stage and type of initial injury. It has been suggested that the different variants of FSG may respond differently to treatment (Valeri. et al. 1996: 1734). Immuno-histochemistry and electron microscopy may also help in differentiating primary and secondary forms of FSGS. It is well known that patients with extensive involvement of glomeruli, advanced tubulo-interstitial fibrosis and tubular atrophy are less likely to respond to corticosteroid treatment compared to those with no interstitial involvement. It is questionable whether it is justifiable to expose such a patient who may also happen to be malnourished and have poor social circumstances to aggressive immune suppression for a disease which may not have an immunological background. The possible departure of many of our black patients from a hostile intra uterine environment resulting in low birth weight and low glomerular endowment is an aspect which has not been investigated systematically.

\section{Conclusions}

Compared to the ISKDC report black children have a lower incidence of MCNS and a higher incidence of FSGS. Black children also have a more aggressive form of FSGS which responds poorly to corticosteroid and other immuno-suppressive treatment. More black children develop CKD stage 4 and 5 compared to white children and black children have a higher mortality compared to white children. The results of this study and similar evidence from the rest of Africa suggest that the ISKDC recommendation of empiric corticosteroid treatment in children with INS should not be followed in the management of black children with INS. We suggest that a kidney biopsy should be done at presentation to allow a definitive diagnosis and targeted treatment from the outset.

\section{References}

Adhikari, M. et al. 1997. Intensive pulse therapies for focal glomerulosclerosis in South African children. Pediatr Nephrol, 11:423-428.

Bhimma, R. 2009. HIV-associated renal disease in children. The Pediatric Quarterly, 1(4), 1518.

Bhimma, R. et al. 2006. Steroid-resistant nephrotic syndrome: the influence of race on cyclophosphamide sensitivity. Pediatr Nephrol, 21, 1847-1853.

Bhimma, R. et al. 1997. Nephrotic Syndrome in South African Children: changing perspectives over 20 years. Pediatr Nephrol, 11, 429-434.

Borges, FF. et al. 2007. Is focal segmental glomerulosclerosis increasing in patients with nephrotic syndrome ? Pediatr Nephrol, 22, 1309-1313.

Filler, G. et al. 2003. Is There Really an Increase in Non Minimal Change Nephrotic Syndrome in Children? Am J Kidney Dis, 42(6), 1107-1114.

Habib, R. \& Kleinknecht, C. 1971. The primary nephrotic syndrome of childhood: Classification and clinicopathologic study of 406 cases. Pathology Annual, 6, 417474.

Hodson, E.M. et al. 2006. Intervention for idiopathic steroid-resistant nephrotic syndrome in children. Cochrane Database Sys Rev, 2, CD003594 
Ingulli, E. \& Tejani, A. 2001. Racial differences in the incidence and renal outcome of idiopathic focal segmental glomerulosclerosis in children. Pediatr Nephrol, 5, 393397.

International Study of Kidney Disease in Children. 1978. Nephrotic syndrome in children: Prediction of histopathology from clinical and laboratory characteristics at the time of diagnosis. A report of the International Study of Kidney Disease in Children. Kidney Int, 13, 159-165.

Kala, U. et al. 1993. Impact of tuberculosis in children with idiopathic nephrotic syndrome. Pediatr Nephrol, 7, 392-395.

K/DOQI clinical practice guidelines for chronic kidney disease: evaluation, classification, and stratification. 2002. Kidney Disease Outcome Quality Initiative. Am J Kidney Dis, 39, S1-S246

Lewin, J.R. et al. 1979. The differing histology in black and white children with nephrotic syndrome (abstract). Kidney Int, 16, 88.

Manalich, R. et al. 2000. Relationship between weight at birth and the number and size of renal glomeruli in humans: a histomorphometric study. Kidney Int, 58, 770773.

Mendoza, S.A. et al. 1990. Treatment of steroid-resistant focal segmental glomerulosclerosis with pulse methylprednisolone and alkylating agents. Pediatr Nephrol, 4, 303-307.

Olowu, W.A. et al. 2010. Reversed Clinical and Morphologic Characteristics of Idiopathic Childhood Nephrotic Syndrome. Int J Nephrol Urol. 2(1), 200 - 211.

Prinsloo, J.G. 1986. The nephrotic syndrome in black children at Kalafong Hospital. SAMJ, 70:375.

Schwartz, G.J. et al. 1987. The use of plasma creatinine concentration for estimating glomerular filtration rate in infants, children and adolescents. Pediatr Clin North Am, 34, 571-590.

Srivastava, T. et al. 1991. High incidence of focal segmental glomerulosclerosis in nephrotic syndrome of childhood. Pediatr Nephrol, 13, 13-18.

Tarshish, P. et al. 1996. Cyclophoshamide does not benefit patients with focal segmental glomerulosclerosis: Report of the International Study of Kidney Diseases in Children. Pediatr Nephrol,10:590-593.

Teeninga, N. et al. 2008. Influence of low birth weight on minimal change nephrotic syndrome in children, including meta-analysis. Nephrol Dial Transplant, 23, 16151620.

The Fourth Report of the National High Blood Pressure Education Program Working Group on High Blood Pressure in Children and Adolescents. 2004. Pediatrics, 114(2), 555575.

Thomson, P.D. 1997. Renal problems in black South African children. Pediatr Nephrol, 1, 508-512.

Valeri, A. et al. 1996. Idiopathic collapsing focal glomerulosclerosis: A clinicopathologic study. Kidney Int, 50, 1734-1746

Vehaskari, V.M. 2007. Developmental origins of adult hypertension: new insights into the role of the kidney. Pediatr Nephrol, 22, 490-495 
White, R.H.R. et al. 1970. Clinicopathologic study of nephrotic syndrome in childhood. Lancet, 1,1353-1359.

Yao Doe, J. et al. 2006. Nephrotic syndrome in African children: lack of evidence of "tropical nephrotic syndrome". Nephrol Dial Transplant, Vol. 21, 672-676. 


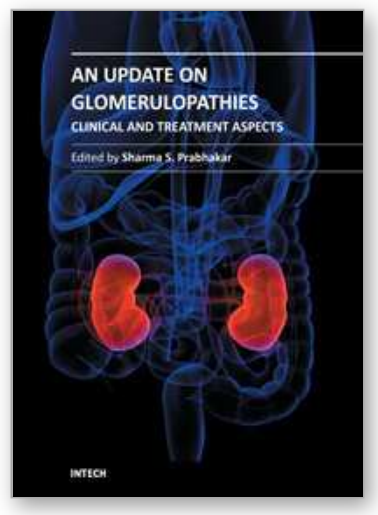

\author{
An Update on Glomerulopathies - Clinical and Treatment Aspects \\ Edited by Prof. Sharma Prabhakar
}

ISBN 978-953-307-673-7

Hard cover, 468 pages

Publisher InTech

Published online 02, November, 2011

Published in print edition November, 2011

An Update on Glomerulopathies - Clinical and Treatment Aspects is a systemic overview of recent advances in clinical aspects and therapeutic options in major syndromes of glomerular pathology. The book contains twenty four chapters divided conveniently into five sections. The first section deals with primary glomerulopathies, and the second section is devoted to glomerulopathies complicating infectious conditions. The third section deals with systemic autoimmune disorders and vasculitides which constitute major causes of glomerular disease and often renal failure. The fourth section includes chapters discussing the glomerular involvement in some major metabolic and systemic conditions. The final section has chapters which relate to some general aspects of glomerular diseases. This book will form an excellent reference tool for practicing and academic nephrology community.

\title{
How to reference
}

In order to correctly reference this scholarly work, feel free to copy and paste the following:

Gertruida van Biljon (2011). Nephrotic Syndrome in Children - Studies from South Africa, An Update on Glomerulopathies - Clinical and Treatment Aspects, Prof. Sharma Prabhakar (Ed.), ISBN: 978-953-307-673-7, InTech, Available from: http://www.intechopen.com/books/an-update-on-glomerulopathies-clinical-andtreatment-aspects/nephrotic-syndrome-in-children-studies-from-south-africa

\section{INTECH}

open science | open minds

\author{
InTech Europe \\ University Campus STeP Ri \\ Slavka Krautzeka 83/A \\ 51000 Rijeka, Croatia \\ Phone: +385 (51) 770447 \\ Fax: +385 (51) 686166 \\ www.intechopen.com
}

\author{
InTech China \\ Unit 405, Office Block, Hotel Equatorial Shanghai \\ No.65, Yan An Road (West), Shanghai, 200040, China \\ 中国上海市延安西路65号上海国际贵都大饭店办公楼 405 单元 \\ Phone: +86-21-62489820 \\ Fax: +86-21-62489821
}


(C) 2011 The Author(s). Licensee IntechOpen. This is an open access article distributed under the terms of the Creative Commons Attribution 3.0 License, which permits unrestricted use, distribution, and reproduction in any medium, provided the original work is properly cited. 